\title{
A Quadrature Modulator Based Scheme for Frequency Hopping Applications
}

\author{
Burak Soker, Sinan Kurt, Gokhun Selcuk \\ HBT-ETM \\ ASELSAN Inc. \\ Ankara, Turkey \\ bsoker,sinank,gselcuk@aselsan.com.tr
}

\begin{abstract}
In this study a new frequency hopping scheme which relies on digital up-conversion of baseband data is proposed. Since the frequency of the local oscillator is kept constant and hopping is achieved in the baseband, the time required for the frequency jump is independent of the frequency step, allowing a narrow loop bandwidth and low noise performance for conventional phase locked loops (PLLs). Moreover the spurious signals resulting from the modulator is around $-70 \mathrm{dBc}$, which is a lower value when compared to spurious signal performance of commercially available direct digital synthesis (DDS) integrated circuits. We have analyzed the error vector magnitude (EVM) and frequency hopping speed on a practical setup and have shown that quite satisfactory results can be obtained with the proposed scheme.
\end{abstract}

Keywords -frequency hopping; digital up-conversion; quadrature modulator; phase locked loop; direct digital synthesis

\section{INTRODUCTION}

Spread spectrum systems are widely utilized in order to overcome performance degradation due to jamming and interference arising from other users. In frequency hopped spread spectrum (FHSS), the data is organized into blocks and each block is transmitted with a different pseudo random carrier frequency enabling redundancy.

In FHSS, no data is transmitted until the carrier frequency is tuned to the next destination frequency which reduces the effective data rate of the whole system. The carrier signal is generated either by using a voltage controlled oscillator (VCO) whose tuning voltage is adjusted within the phase locked loop (PLL) or a direct digital frequency synthesizer (DDFS), which generates the carrier signal with a digital to analog converter (DAC) according to the frequency tuning word.

In applications where conventional PLL's are used, the frequency hopping speed is primarily determined by loop dynamics. The time required for a PLL to lock to a target frequency is given by [1],

$$
t_{\text {lock }}=\frac{-\ln \left(\sqrt{1-\xi^{2}} \Delta f / f_{\text {tol }}\right)}{\xi \omega_{n}}
$$

where $\xi$ is the damping factor, $\omega_{n}$ is the closed loop bandwidth, $\Delta f$ is the frequency step and $f_{\text {tol }}$ is the tolerance around the destination frequency. In order to increase the settling speed, the loop bandwidth can be increased but this will result in degradation in the phase noise performance of the PLL affecting the ultimate error vector magnitude (EVM) of the whole system. Moreover, the settling time is a function of the frequency step and in the design phase the maximum frequency step should be used as one of the design criteria. Although studies are conducted to alleviate the difficulty regarding the settling time [2-4], significant performance improvements could not be obtained without an additional effort.

An alternative solution to conventional PLLs is to generate the carrier signals digitally by using commercially available DDFS integrated circuits. As the carrier signal is digitally produced, the time required for a frequency jump is limited by nothing but the register writing speed, therefore DDFS is much faster than the conventional PLL architecture [5]. However it has two main disadvantages. The first one is that, the maximum frequency that a DDFS can synthesize is limited upto only a few $\mathrm{GHz}$, making their use impractical for high frequency applications, unless a hybrid approach, which includes the use of PLL as well, is utilized. The other main drawback of DDFS is that, digital generation of carrier signals introduces spurious signals as strong as $-50 \mathrm{dBc}$ which makes it difficult to satisfy spectrum mask requirements [6].

In the present study, we propose a novel scheme based on digital up-conversion of the baseband data, which is used to modulate a quadrature modulator. As the frequency hopping is performed at the baseband, the maximum frequency jump is limited by the sampling frequency of the DAC. Considering the performance of the commercially available DACs, the proposed method is suitable for systems with sub-GHz frequency jumps. The local oscillator for the modulator is supplied from a conventional PLL with narrow loop bandwidth; hence there is no contribution to EVM from the carrier signal. The carrier leakage and the sideband suppression of the modulator can be calibrated such that they are both lower than $-70 \mathrm{dBc}$, which is considered as an improvement over DDFS systems.

We have tested the proposed scheme on a practical setup and obtained satisfactory results in terms of frequency hopping speed and EVM. The hopping speeds on the order of nanoseconds and the EVM values less than $\% 1$ are observed over the whole bandwidth. 


\section{THE PRoposed SCHEME}

Block diagram of the proposed scheme, namely digitally up-converted IQ data generation, is illustrated in Fig. 1.

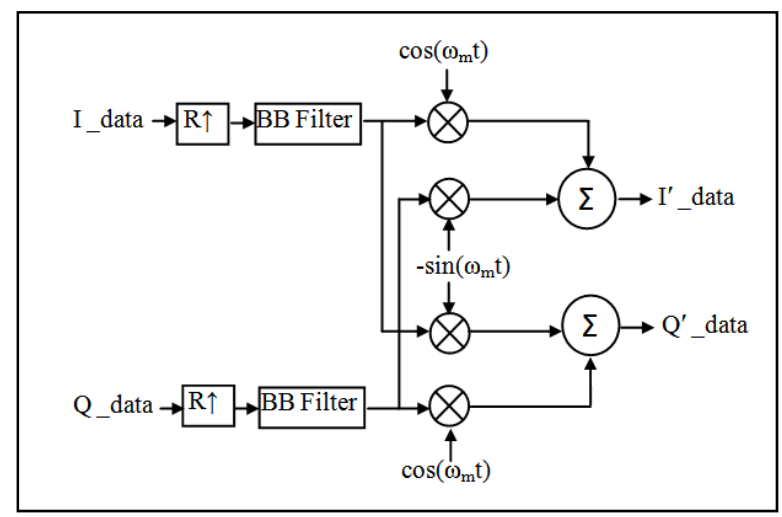

Figure 1. Generation of digitally up-converted data

As shown in Fig. 1, the frequency jump is achieved using a full complex mixer and a numerical oscillator (NO) at frequency $\omega_{\mathrm{m}}$. The new in phase and quadrature signals are generated according to,

$$
\begin{gathered}
I_{\text {data }}^{\prime}(t)=I_{\text {data }}(t) \cos \left(\omega_{m} t\right)-Q_{\text {data }}(t) \sin \left(\omega_{m} t\right) \\
Q_{\text {data }}^{\prime}(t)=Q_{\text {data }}(t) \cos \left(\omega_{m} t\right)-I_{\text {data }}(t) \sin \left(\omega_{m} t\right)
\end{gathered}
$$

where $\omega_{\mathrm{m}}$ is the frequency of the NO and the primed data variables refer to the digitally up-converted data.

Despite the ideal multiplication as depicted in Fig. 1, some detrimental factors degrade the performance of this system. Since IQ signals are up-converted in the baseband before conventional mixing, the DAC can not be operated at the same frequency as if there is no up-conversion. Not only the bandwidth but also the frequency shift of the IQ data should be considered while selecting the minimum sampling frequency of the DAC. The DAC sampling frequency can be written as,

$$
f_{s}>2 \cdot(\Delta f+W)
$$

where $f_{s}$ is the sampling frequency, $\pm \Delta f$ is the maximum frequency jump and $\mathrm{W}$ is the bandwidth of the signal. Note that, the generated baseband signal has a bandwidth $\mathrm{W}$, before frequency shift. Therefore, in this equation $\mathrm{W}$ is used as the one-sided bandwidth.

Use of high frequency DACs instead of their low frequency counterparts is undesirable when power consumption, noise performance and harmonic distortion parameters are considered. Due to the necessity of the use of a high speed DAC, the phase noise performance at the output of the modulator will be determined by the NO. High quantization noise of the DAC will dominate the total phase noise of the modulator. It should be noted that the sampling frequency limited by (3), is much less than the sampling frequency of the DACs employed in DDS circuits. Therefore the method proposed in this study will lead to improved results in terms of phase noise performance and harmonic distortion when compared to systems employing DDS in their architectures.
Apart from the degradations introduced by high speed DACs, some spurious content is also introduced by the imbalance of the quadrature modulator. The phase/amplitude imbalance between the in-phase and quadrature paths will result in some carrier leakage and sideband signals to be observed at the output spectrum of the modulator. Given the phase imbalance $\theta$ in degrees and the amplitude imbalance $\alpha$ in $\mathrm{dB}$, the sideband signal level (SSL) in dBc's is given by [7],

$$
\begin{gathered}
S S L_{\text {phase.imb. }}=20 \log \cot (\theta / 2) \\
S S L_{\text {amp.imb. }}=20 \log \left(\frac{10^{\alpha / 20}-1}{10^{\alpha / 20}+1}\right)
\end{gathered}
$$

Since the frequency of the transmitted data and the local oscillator signal do coincide, these spurious signals in $(4,5)$ will appear outside the bandwidth allocated for the channel, possibly violating spectrum mask requirements. Moreover as the frequency of the $\mathrm{I} / \mathrm{Q}$ signals is increased, the system is less immune to differences due to path difference between the transmission line paths of $\mathrm{I} / \mathrm{Q}$ signals. Also the specifications of both I/Q modulator ICs and DACs degrade at high speeds, deteriorating spur performance. Although the IQ imbalance may seem a serious problem for the proposed scheme, it should be noted that the detrimental effects due to the imbalance can be compensated by proper calibration of the phase/amplitude of the baseband signals. Undesired spurious signals can be decreased to about $-70 \mathrm{dBc}$, which is a satisfactory value when compared to spurious performance of commercially available DDS components.

\section{PRACTICAL IMPLEMENTATION}

In order to test the performance of the proposed scheme, we used a practical setup whose block diagram is shown in Fig. 2.

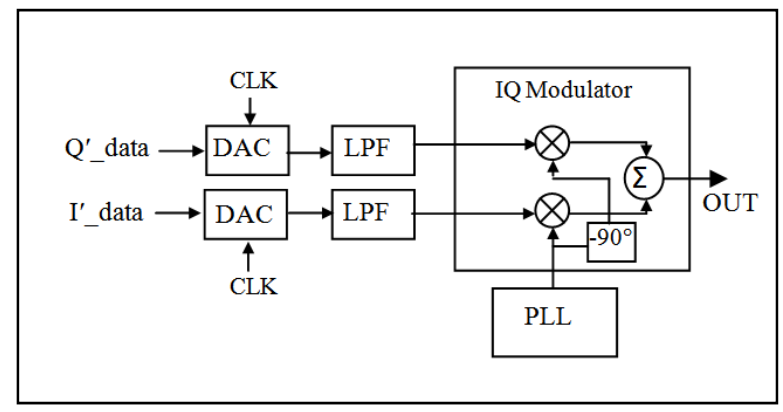

Figure 2. Block diagram of the setup.

The digitally generated data is fed to $100 \mathrm{Mbps} 16-$ bit DACs internal to E4438C signal generator. The outputs of the DACs are filtered using a $40 \mathrm{MHz}$ lowpass filter to eliminate aliasing terms from the DAC. The filtered baseband signal is fed to the quadrature modulator. The quadrature modular used in the setup is BGX7101 from NXP which supports wide modulation bandwidth $(650 \mathrm{MHz})$. The local oscillator for the modulator is supplied from another signal generator with low phase noise. 
The phase noise at the output of the modulator is determined by both the PLL and the DAC. In order to test the phase noise performance of the scheme we generated unmodulated signals at different offsets from the PLL frequency. The phase noise measurements are plotted in Fig. 3.

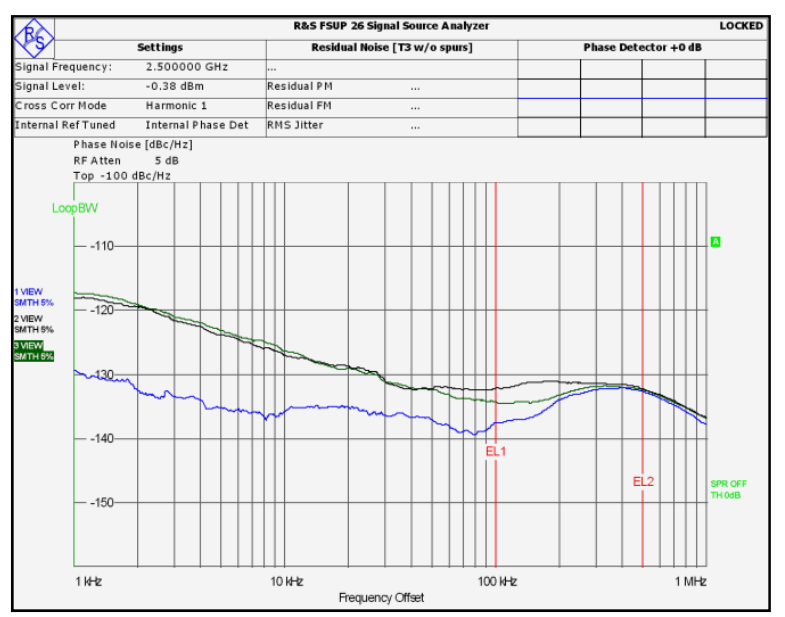

Figure 3. Phase noise at the output of the modulator

The blue curve in Fig. 3 plots the phase noise performance of the PLL which is operated at $2.5 \mathrm{GHz}$. As for the green and black curve measurements, IQ modulator output is digitally shifted $1 \mathrm{MHz}$ and $25 \mathrm{MHz}$ respectively. Therefore, using the same PLL frequency, namely $2.5 \mathrm{GHz}$, the green and black curves show the phase noise performance at the frequencies $2.501 \mathrm{GHz}$ and $2.525 \mathrm{GHz}$. Since green and black curves result in similar phase noise plots, we conclude that the clock phase noise of the DAC is negligible and the main contributors are the quantization noise and thermal noise of DAC.

Next we observed the spurious performance of the proposed scheme. In Fig. 4 the output spectrum of the IQ modulator is plotted with LO frequency adjusted to $2.5 \mathrm{GHz}$. The baseband signals are generated such that a sine wave at frequency $2.525 \mathrm{GHz}$ is observed at the output of the modulator.

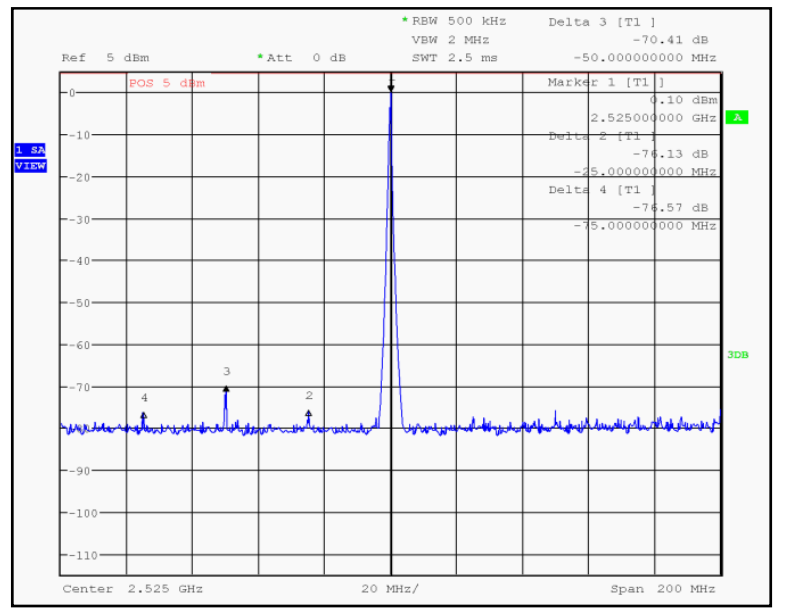

Figure 4. Spur performance of the modulator.

In Fig 4 two types of spurious signals are observed. The spurs at $2.5 \mathrm{GHz}$ and $2.475 \mathrm{GHz}$ are the carrier feed through and the sideband signals due to the imbalance of the baseband data. These spurs have the amplitudes of $-76.14 \mathrm{dBc}$ and $-70.4 \mathrm{dBc}$ respectively. The spurious signal at $2.45 \mathrm{GHz}$ results from the nonlinearity of the DAC and is $76.57 \mathrm{~dB}$ below the carrier. Due to the low-speed operation of the DAC, its spurious free dynamic range is higher. Therefore the main contributor to the spurious performance in this scheme is signals arising from the modulator imperfections. It should be noted that the spurious performance of this scheme is about $70 \mathrm{dBc}$, which can be considered as a quite satisfactory result outperforming commercially available DDS-ICs operating at $\mathrm{GHz}$ frequencies.

In order to investigate the detrimental effects due to the digital up conversion, we have also generated a QPSK signal. The baseband signal is up-converted above $25 \mathrm{MHz}$ of the carrier frequency. EVM is measured about $0.3 \%$, which is the lower limit of the measuring device. It can be deduced that the digital up-conversion does not deteriorate the EVM performance. The constellation diagram and the measured values are given in Fig. 5.

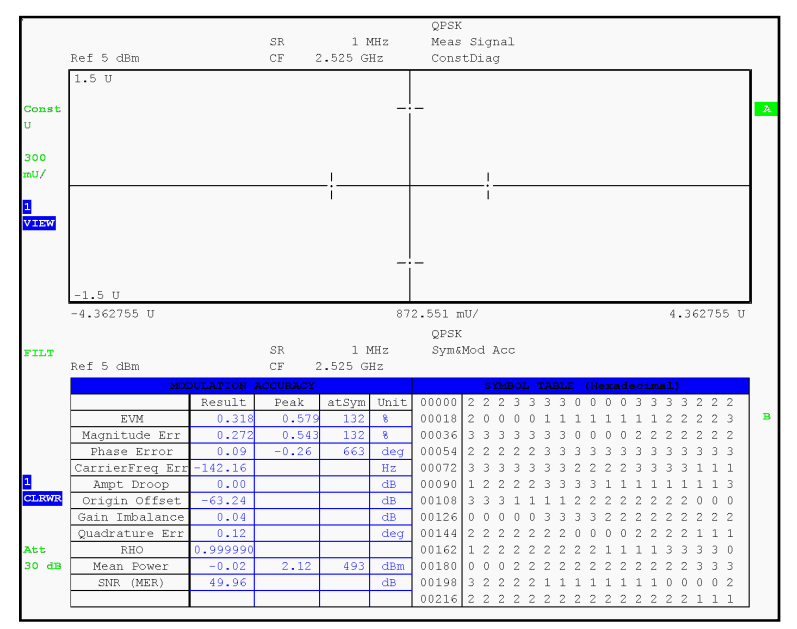

Figure 5. The constellation diagram for the QPSK signal

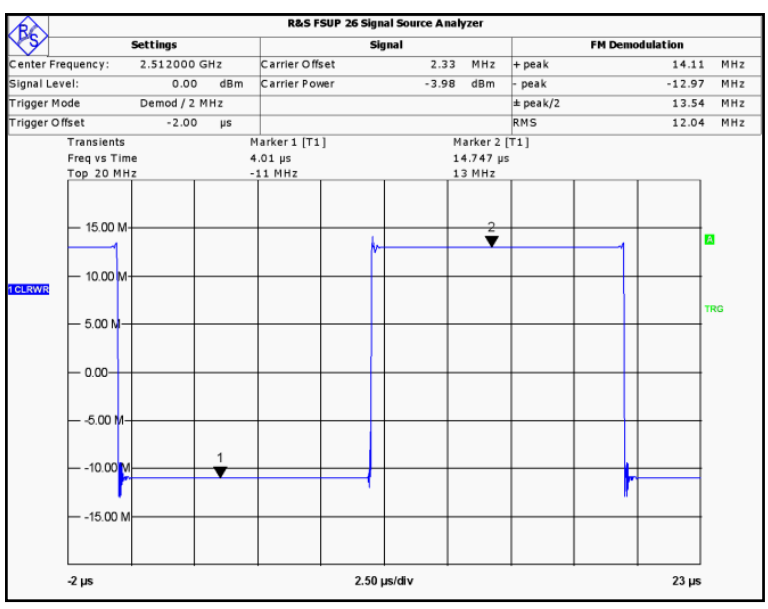

Figure 6. Frequency transition in the proposed scheme

Finally we investigated the frequency settling time of the scheme by sequentially generating two baseband signals leading to different carrier frequencies at the output of the modulator. The 
frequency jump from $2.525 \mathrm{GHz}$ to $2.501 \mathrm{GHz}$ is shown in Fig. 6.

The fluctuations seen at the edges of the frequency transitions in Fig. 6 are due to the glitches generated by the DAC. These glitches are filtered by the $30 \mathrm{MHz}$ bandwidth of the measuring device. Due to the limited bandwidth of the measuring device, the settling time could not be obtained accurately. However we can deduce that the settling time is on the order of a few hundred nanoseconds. The detailed frequency transition plot is given in Fig. 7.

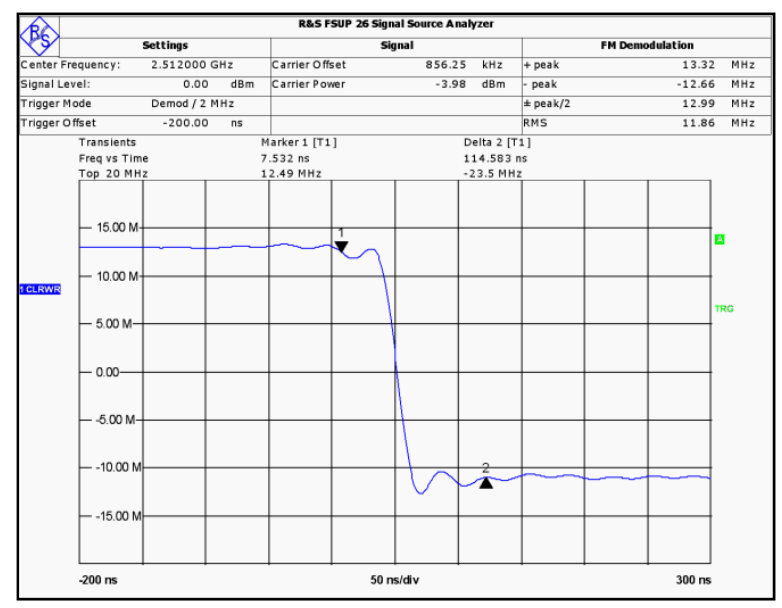

Figure 7. Frequency transition enlarged

\section{CONCLUSIONS}

In this study, we propose a novel method to generate frequency hopping signals using a quadrature modulator. Instead of hopping the carrier frequency of the quadrature modulator, frequency change is achieved by digital up-conversion of the baseband data. Using this method, the requirement of the DAC specifications is considerably reduced as compared to the DACs employed in DDS circuits. This is because the maximum speed of the DACs is determined by not maximum operating frequency but maximum offset needed for the frequency hop. Because of the lowfrequency operation of the DAC in the proposed scheme, both quantization noise and SFDR are improved leading to lower phase noise and better dynamic range. Moreover the settling time, which is constrained by the loop dynamics of the PLLs, is no more of concern. In the proposed scheme, the frequency hop can be achieved instantly as in the case of DDS-ICs. Furthermore, spurious levels are not limited by DAC performance but rather modulator spurs and can be decreased severely by proper calibration of the baseband IQ signals. The spur levels measured in the proposed scheme are lower compared to the high-speed DACs within DDS-ICs.

\section{REFERENCES}

[1] A. Cicero, Architectures for RF Frequency Synthesizers, Kluwer Acad. Pub., New York, US, 2003.

[2] X. F. Kuang and N. J. Wu, "A fast-settling monolithic PLL frequency synthesizer with direct frequency presetting," in ISSCC Dig. Tech. Papers, pp. 204-205, pp. 855-865, Feb. 2006,

[3] A. Zhang, P. E. Allen, J. M. Huard, " A fast switching PLL frequency synthesizer with an on-chip passive discrete-time loop filter in 0.25 um CMOS," IEEE J. Solid-State Circuits, vol.38, no. 6, Jun. 2003

[4] K. Woo, Y.liu, E.Nam and D. Ham, "Fast-lock hybrid PLL combining fractional-N and integer- $\mathrm{N}$ modes of differing bandwidths," IEEE J. Solid-State Circuits, vol. 43, no. 2, pp. 379-389, Feb. 2008

[5] J. Vankka, "Digital frequency synthesizer/modulator for continious-phase modulations for slow frequency hopping," in IEEE Trans .on Veh. Tech., vol.38, pp. 933-940, no. 4, Nov. 1997

[6] K. Kwon, W. Yoon, “ Methods to supress DDFS spurious signals in a frequency-hopping synthesizer with DDFS-driven PLL architecture," in IEEE Trans. on Ultr Ferro. Freq. Cont. , vol.57, pp. 299-304, no. 2, Feb. 2010

[7] C. L. Liu, "Impacts of I/Q imbalance on QPSK-OFDM-QAM detection”, IEEE Trans. Cons. Electr., vol. 44, no 3, pp 984989, Aug. 1998 